\title{
DEEP LEARNING FOR EARTHQUAKE DETECTION AND LOCATION
}

\section{Saumik Dana ${ }^{1, *}$}

\author{
${ }^{1}$ University of Southern California, Los Angeles, CA 90089 \\ *Address all correspondence to: Saumik Dana, E-mail: sdana@usc.edu
}

Understanding the causality between the events leading to fault slip and the earthquake recording is important for seismic design and monitoring of underground structures, bridges and reinforced concrete buildings as well as climate mitigation projects like carbon sequestration and energy technologies like enhanced geothermal systems or oilfield wastewater disposal. The Federal Emergency Management Agency (FEMA) reported in 2017, that earthquake losses in the United States add up to about $\$ 6.1$ billion dollars annually. This number only addresses direct economic losses to buildings, and does not cover damage and losses to critical facilities, transportation and utility lifelines or indirect economic losses. A holistic framework to study earthquakes would incorporate seismic wave propagation and pressure perturbations, and have a dialogue with the deep learning framework for earthquake detection and location. In this document, we delve into the deep learning module.

KEY WORDS: Earthquakes, deep learning

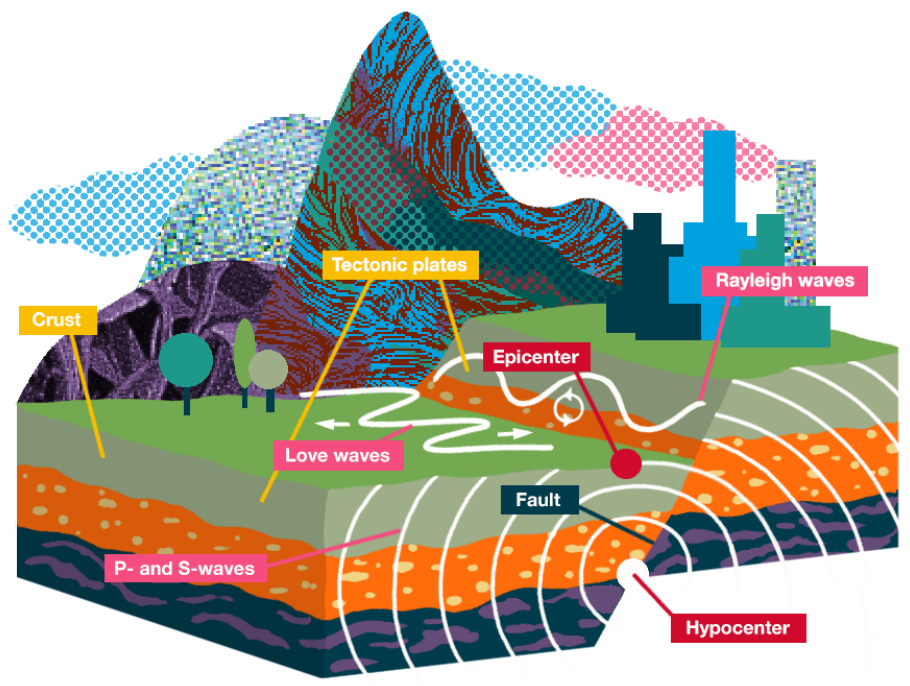

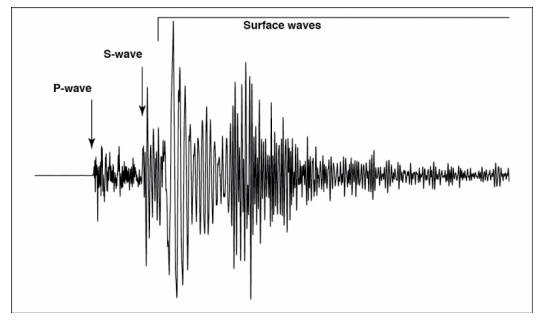

(b) P-, S- and surface waves

(a) Propagation of waves

FIG. 1: Source: Caltech Science Exchange

The earthquake cycle, from slow deformation associated with interseismic behavior to rapid deformation associated with earthquake rupture, spans spatial scales ranging from fractions of a meter associated with the size of contact asperities on faults and individual grains to hundreds of kilometers associated with plate boundaries (Kanamori and Brodsky (2004)). Similarly, temporal scales range from fractions of a second associated with slip at a point during 


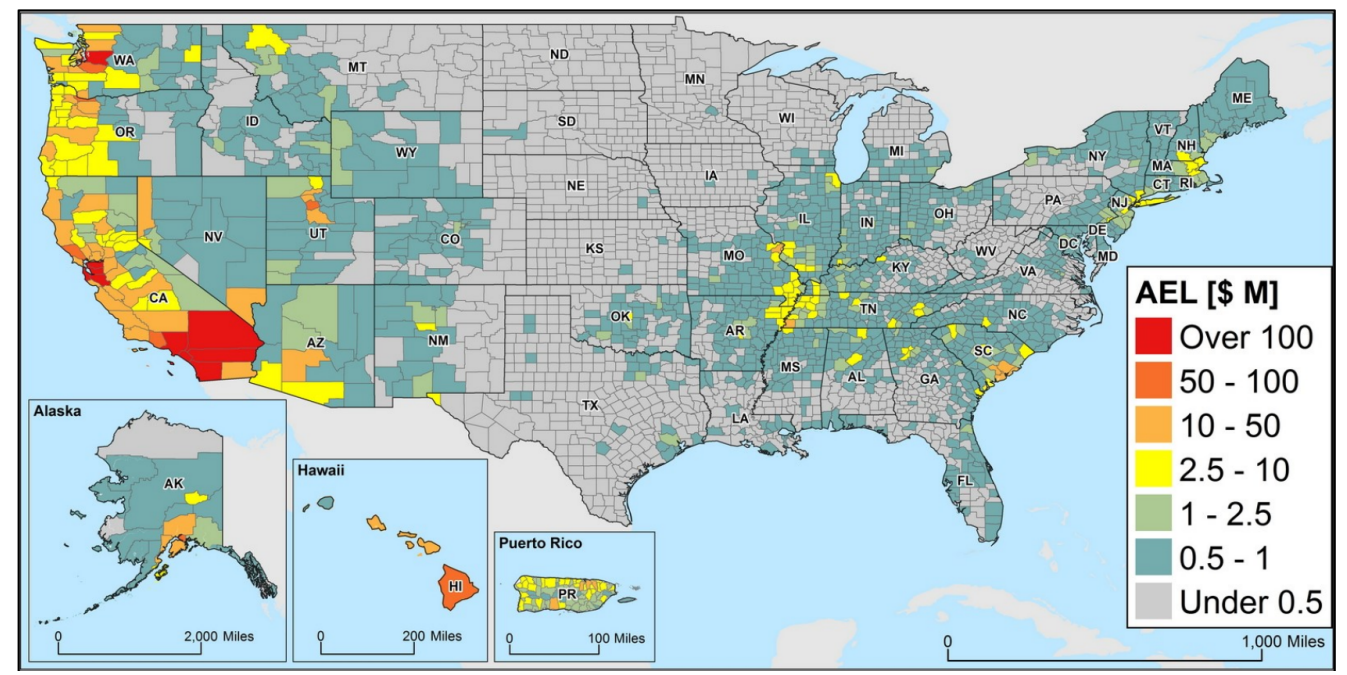

FIG. 2: Annualized Earthquake Losses (AEL: estimated long-term value of earthquake losses to the general building stock) by County. Sources: (1) https://www.usgs.gov/news/usgs-collaborates-fema-national-earthquake-loss-estimate and (2) https://www.fema.gov/hazus. The analysis yielded an estimate of the national AEL of $\$ 6.1$ billion per year. Loss estimates based on the best science and engineering available (during 2016-2017). The study only addresses direct economic losses to buildings, and does not cover damage and losses to critical facilities, transportation and utility lifelines or indirect economic losses.

earthquake rupture to hundreds of years of strain accumulation between earthquakes. In many cases, earthquakes are triggered after pore pressure perturbations activate critically stressed seismogenic faults (Kanamori and Brodsky (2004)), not just due to natural causes like earth tides (Scholz et al. (2019)), rainfall (Hainzl et al. (2006)), snowfall (Montgomery-Brown et al. (2019)), typhoons (Liu et al. (2009)), but also due to human activity (Foulger et al. (2018)). As faults slip, the induced stress field spawns seismic waves as shown in Fig. 1a, which travel through and around the earth and can be recorded with seismometers as shown in Fig. 1b. Ground motion and failure due to earthquakes lead to huge losses to the tune of billions of dollars annually in the United States (see Fig. 2). Understanding the causality between the events leading to fault slip, the location of the slipping fault and the earthquake recording is important for seismic design and monitoring of underground structures (Hashash et al. (2001)), bridges (Priestley et al. (1996)) and reinforced concrete buildings (Moehle (2015)) as well as climate mitigation projects like carbon sequestration (Zoback and Gorelick (2012)) and energy technologies like enhanced geothermal systems (Ellsworth et al. (2019)) or oilfield wastewater disposal (Keranen and Weingarten (2018)).

Most earthquake detection methods are designed for moderate and large earthquakes. As a consequence, they tend to miss many of the low-magnitude earthquakes that are masked by seismic noise. Detecting and cataloging these earthquakes are key to understanding their causes (natural or human-induced) and, ultimately, to mitigating the seismic risk. Although template matching techniques (which leverage the bank of seismic fingerprints to characterize the seismogram output) have seen a lot of advances in the past decade (Yoon et al. (2015)), they still suffer from poor earthquake location estimates. On the other hand, artificial neural network based techniques offer huge promise in both earthquake detection and probabilistic hypocenter location estimates (Perol et al. (2018); Zhang et al. (2020)). A holistic framework to study earthquakes would incorporate seismic wave propagation and subsurface pressure perturbations, and have a dialogue with the deep learning framework for earthquake detection and location. The first step is rendering quasi-static simulations (Dana (2018, 2019); Dana et al. (2018, 2020); Dana and Jha (2021); Dana and Wheeler (2018a,b, 2019); Dana et al. (2021a,b); Jha and Juanes (2014); Jin and Zoback (2018)) in which wave propagation is ignored and the solution is a time series of solutions to static problems with potentially time-varying physical properties and boundary conditions. The next step is to leverage this subject matter expertise to render fully dynamic simulations. The final step is to deploy deep learning to arrive at hypocenter estimates from seismogram signals and/or satellite images. 


\subsection{Deep learning applied to seismogram signals}

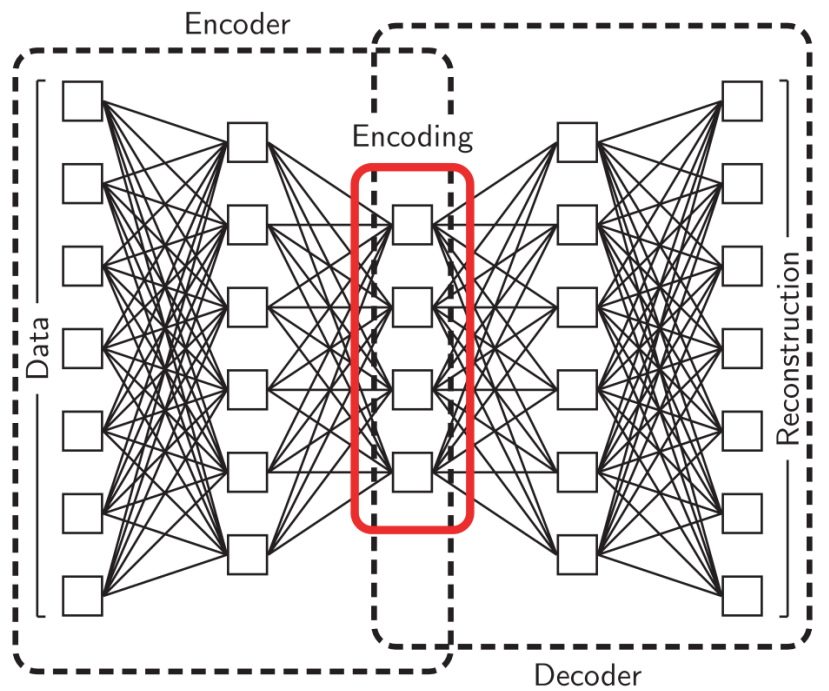

FIG. 3: Architecture of an autoencoder network. Successive layers of nodes detect patterns in input data, and use these to generate an encoded representation of the data. The corresponding decoder takes encodings, and attempts to reconstruct the original inputs. Source: Valentine and Trampert (2012)

Dimensionality reduction facilitates the classification, visualization, communication, and storage of highdimensional data. A simple and widely used method is principal components analysis (PCA), which finds the directions of greatest variance in the data set and represents each data point by its coordinates along each of these directions. A nonlinear generalization of PCA uses an adaptive, multilayer "encoder" network to transform the highdimensional data into a low-dimensional code and a similar "decoder" network to recover the data from the code (Hinton and Salakhutdinov (2006), see Figs. 3, 4 and 5).

\subsection{Deep learning applied to satellite images}

Early attempts at solving inverse imaging problems using convolutional neural networks (Zhou et al. (1988)) were largely superseded by compressed sensing (or, broadly, convex optimization with regularization) approaches in the 2000s. As computer hardware improved, it became feasible to train larger neural networks, until, in 2012, Krizhevsky et al. (2012) achieved a significant improvement over the state of the art on the ImageNet classification challenge by using a graphics processing unit (GPU) to train a $\mathrm{CNN}$ with five convolutional layers and 60 million parameters on a set of 1.3 million images. This work spurred a resurgence of interest in neural networks and, specifically, CNNs-not only for computer vision tasks but also for inverse problems.

Similar to solving inverse problems in medical imaging (Fig. 6), fully convolutional networks can be used to predict a 3D image of the earthquake location probability in the Earth from a volume of input data recorded at multiple network seismic stations (Zhang et al. (2020)). Due to the underlying physics, there is a nonlinear relationship between the seismogram data and event location parameters in an earthquake location problem. Accordingly, the model outputs a 3D image volume that represents the probability of the event location in the subsurface. The point within the image with the largest magnitude marks the most likely event location (Fig. 7). 

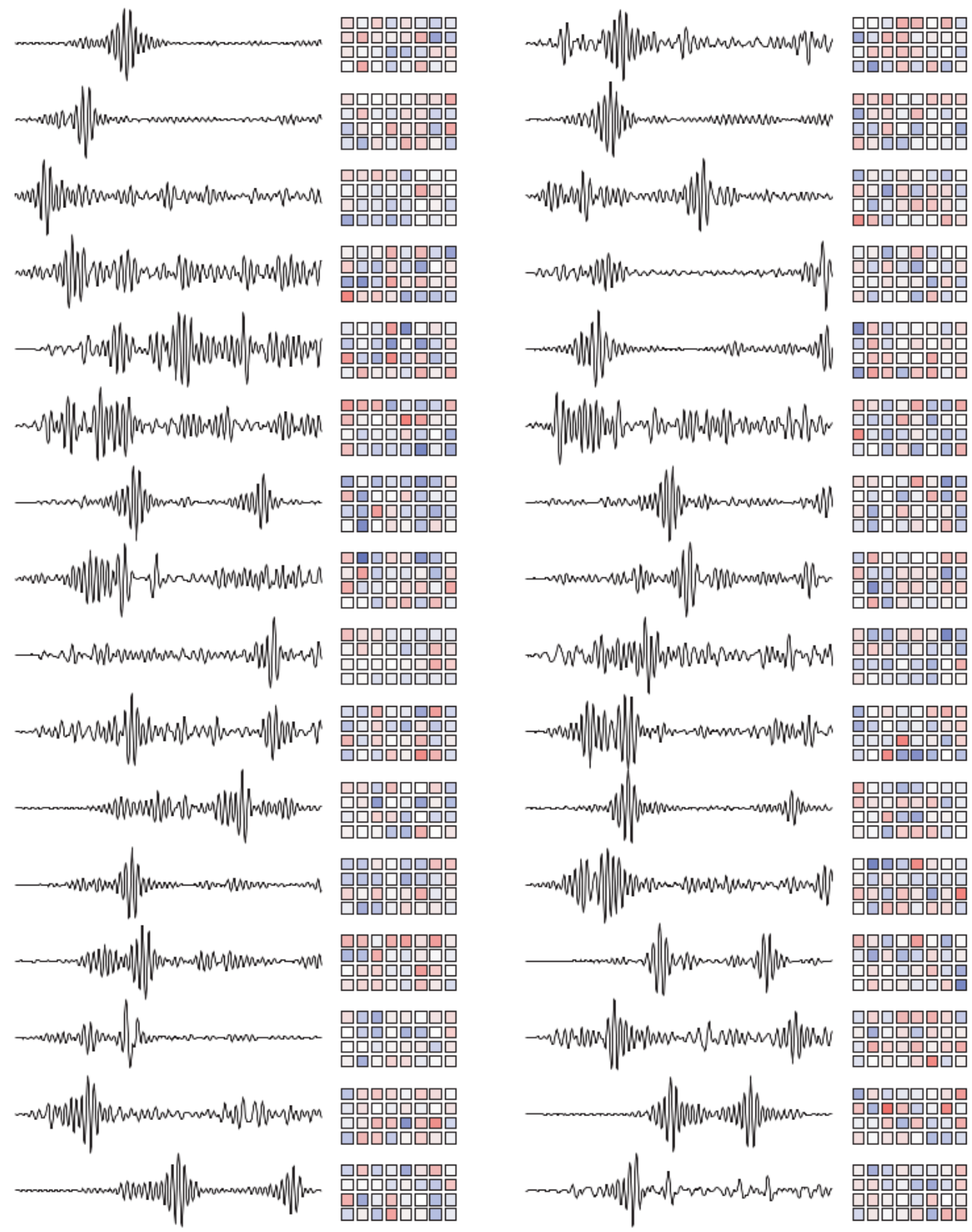

FIG. 4: Encoding waveforms. Red colours represent positive numbers, and blues are negative. Source: Valentine and Trampert (2012) 
枵枵骂㗊

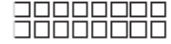

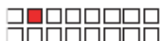

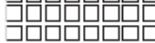

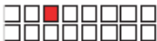
彄骂骂颚

强吅吅吅 彄㗊㗊品

抲品鹃骂

品品骂骂

吅吅吅

强品品呴

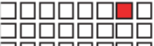

彄㗊㗊

炽吅吅煰

彄呮呮

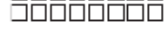

彄吅吅吅

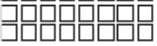

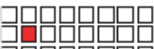

彄㗊㗊

炽吅吅

弱㗊㗊颚

(1)

彄吅吅吅

彄澏湢

गप्रण

彄品吅吅

彄㗊骂

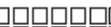

的的哟

彄骂骂煰

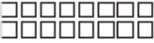

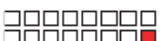

彄熍㗊品

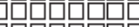

吅吅响

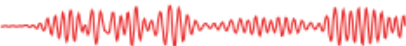

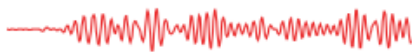

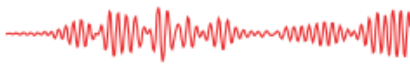

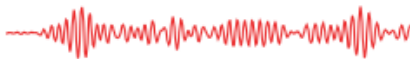

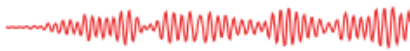

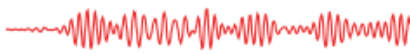

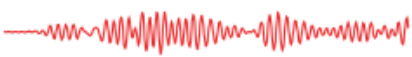

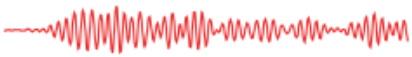

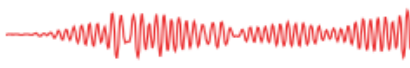

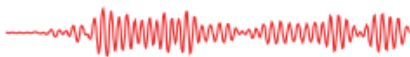

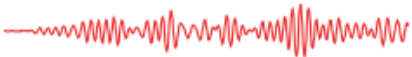

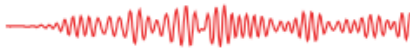

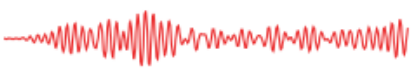

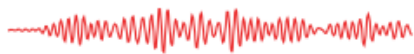

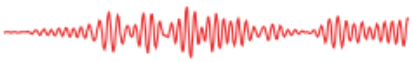

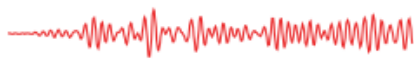

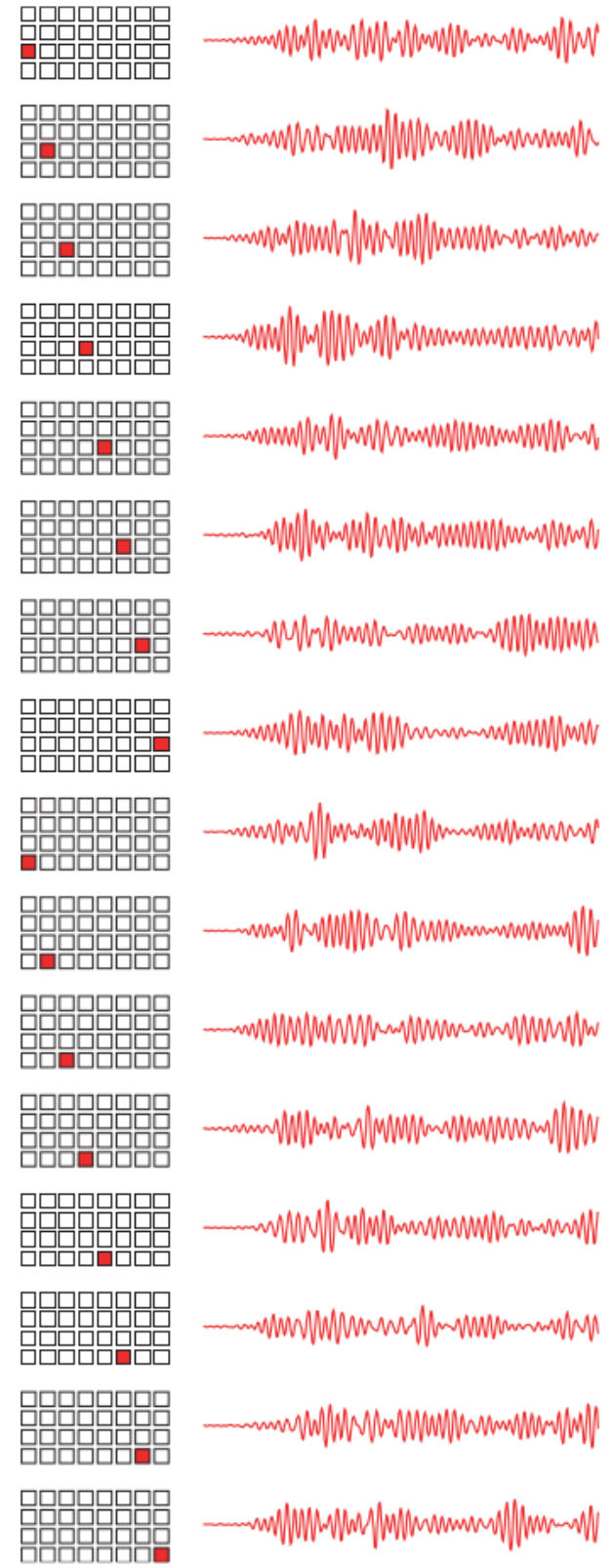

FIG. 5: Decoding. Waveforms corresponding to unit encodings. Source: Valentine and Trampert (2012) 


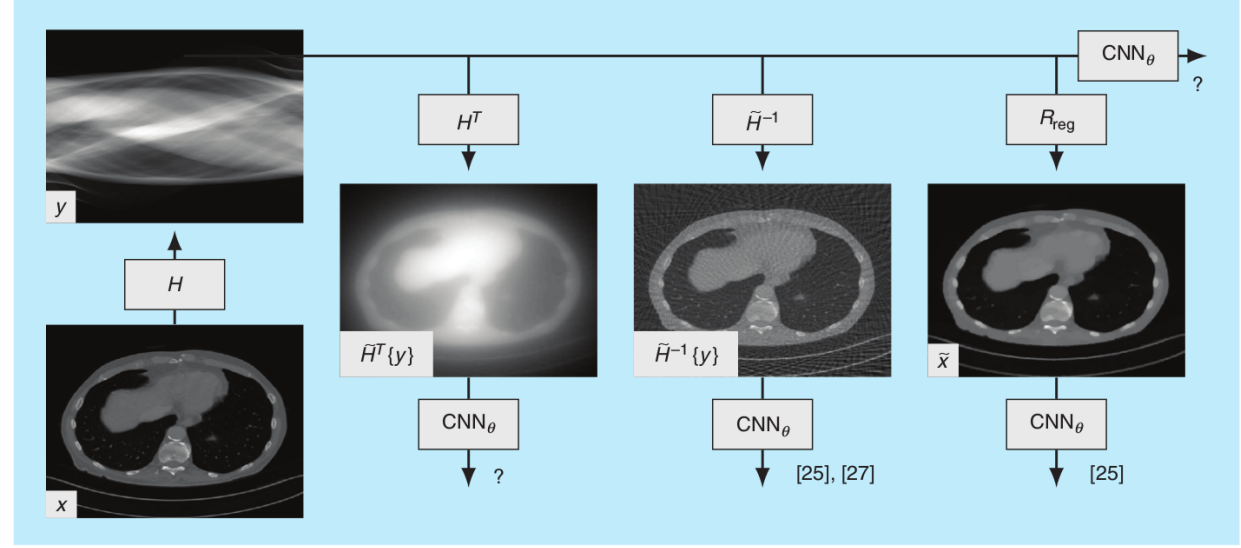

FIG. 6: A block diagram of image reconstruction methods, using images from X-ray CT as examples. An image, $x$, creates measurements, $y$, that can be used to estimate $x$ in a variety of ways. The traditional approach is to apply a direct inversion, which is artifact prone in the sparse-measurement case (note the stripes in the reconstruction). Several recent works apply CNNs to the result of the direct inversion or an iterative reconstruction, but it might also be reasonable to use as input the measurements themselves or the back projected measurements. Source: McCann et al. (2017) 


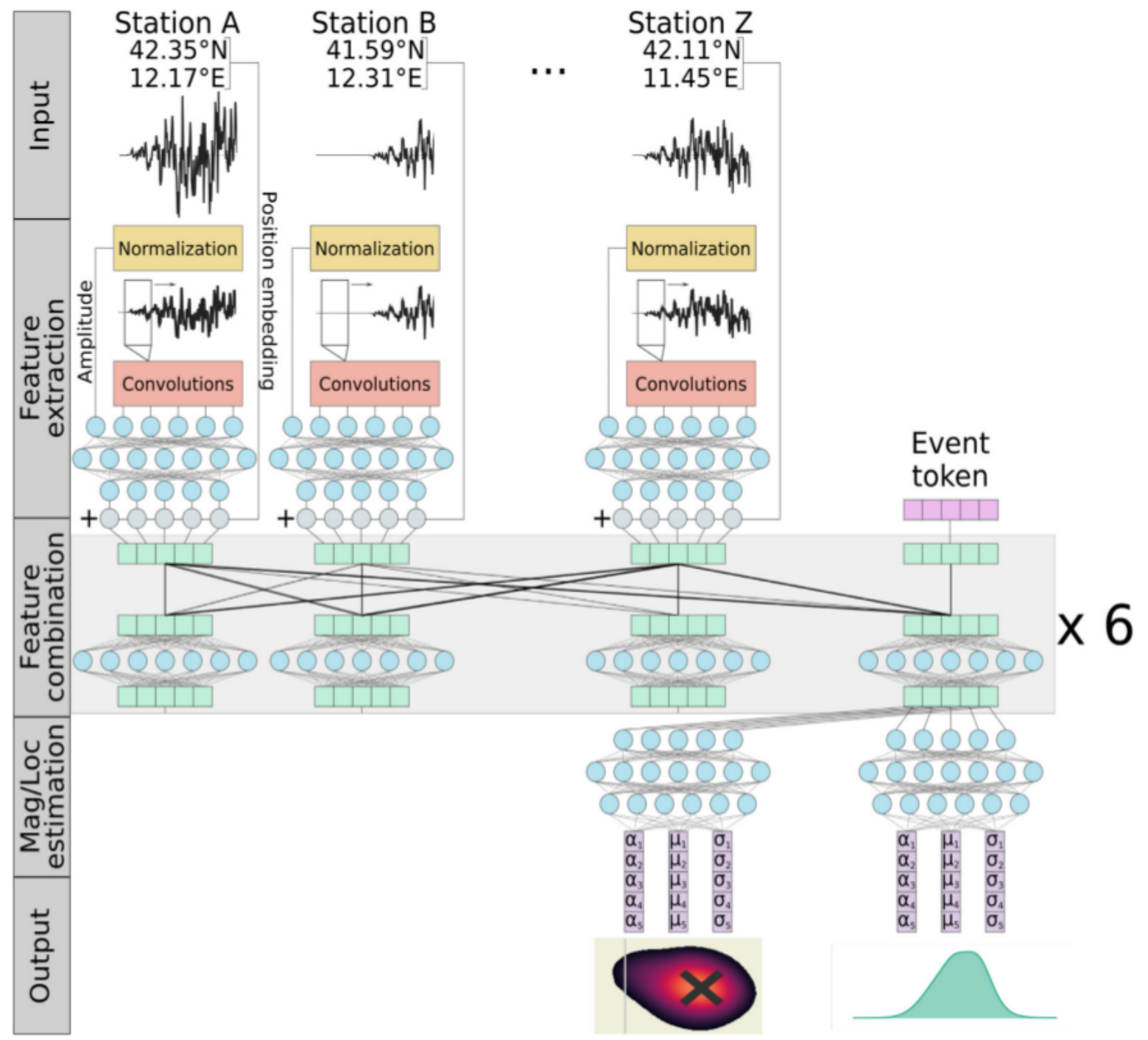

FIG. 7: Input, the feature extraction, the feature combination, the magnitude/location estimation and the output. Source: Münchmeyer et al. (2021) 


\section{References}

Dana, S., Addressing challenges in modeling of coupled flow and poromechanics in deep subsurface reservoirs, PhD thesis, The University of Texas at Austin, 2018.

Dana, S., System of equations and staggered solution algorithm for immiscible two-phase flow coupled with linear poromechanics, arXiv preprint arXiv:1912.04703, 2019.

Dana, S., Ganis, B., and Wheeler, M.F., A multiscale fixed stress split iterative scheme for coupled flow and poromechanics in deep subsurface reservoirs, Journal of Computational Physics, vol. 352, pp. 1-22, 2018.

Dana, S., Ita, J., and Wheeler, M.F., The correspondence between voigt and reuss bounds and the decoupling constraint in a twogrid staggered algorithm for consolidation in heterogeneous porous media, Multiscale Modeling \& Simulation, vol. 18, no. 1, pp. 221-239, 2020 .

Dana, S. and Jha, B., A fault slip model to study earthquakes due to pore pressure perturbations, arXiv preprint arXiv:2104.06257, 2021.

Dana, S. and Wheeler, M.F., Convergence analysis of fixed stress split iterative scheme for anisotropic poroelasticity with tensor biot parameter, Computational Geosciences, vol. 22, no. 5, pp. 1219-1230, 2018a.

Dana, S. and Wheeler, M.F., Convergence analysis of two-grid fixed stress split iterative scheme for coupled flow and deformation in heterogeneous poroelastic media, Computer Methods in Applied Mechanics and Engineering, vol. 341, pp. 788-806, $2018 \mathrm{~b}$.

Dana, S. and Wheeler, M.F., Design of convergence criterion for fixed stress split iterative scheme for small strain anisotropic poroelastoplasticity coupled with single phase flow, arXiv preprint arXiv:1912.06476, 2019.

Dana, S., Zhao, X., and Jha, B., A two-grid computational framework for fast monitoring of fault stability and ground deformation in multiphase geomechanics, Under review at Journal of Computational Physics, 2021a.

Dana, S., Zhao, X., and Jha, B., Two-grid method on unstructured tetrahedra: Applying computational geometry to staggered solution of coupled flow and mechanics problems, arXiv preprint arXiv:2102.04455, 2021b.

Ellsworth, W.L., Giardini, D., Townend, J., Ge, S., and Shimamoto, T., Triggering of the pohang, korea, earthquake (m w 5.5) by enhanced geothermal system stimulation, Seismological Research Letters, vol. 90, no. 5, pp. 1844-1858, 2019.

Foulger, G.R., Wilson, M.P., Gluyas, J.G., Julian, B.R., and Davies, R.J., Global review of human-induced earthquakes, EarthScience Reviews, vol. 178, pp. 438-514, 2018.

Hainzl, S., Kraft, T., Wassermann, J., Igel, H., and Schmedes, E., Evidence for rainfall-triggered earthquake activity, Geophysical Research Letters, vol. 33, no. 19, 2006.

Hashash, Y.M., Hook, J.J., Schmidt, B., John, I., and Yao, C., Seismic design and analysis of underground structures, Tunnelling and underground space technology, vol. 16, no. 4, pp. 247-293, 2001.

Hinton, G.E. and Salakhutdinov, R.R., Reducing the dimensionality of data with neural networks, science, vol. 313, no. 5786, pp. 504-507, 2006.

Jha, B. and Juanes, R., Coupled multiphase flow and poromechanics: A computational model of pore pressure effects on fault slip and earthquake triggering, Water Resources Research, vol. 50, no. 5, pp. 3776-3808, 2014.

Jin, L. and Zoback, M.D., Fully dynamic spontaneous rupture due to quasi-static pore pressure and poroelastic effects: An implicit nonlinear computational model of fluid-induced seismic events, Journal of Geophysical Research: Solid Earth, vol. 123, no. 11, pp. 9430-9468, 2018.

Kanamori, H. and Brodsky, E.E., The physics of earthquakes, Reports on Progress in Physics, vol. 67, no. 8, p. $1429,2004$.

Keranen, K.M. and Weingarten, M., Induced seismicity, Annual Review of Earth and Planetary Sciences, vol. 46, pp. 149-174, 2018. 
Krizhevsky, A., Sutskever, I., and Hinton, G.E., Imagenet classification with deep convolutional neural networks, Advances in neural information processing systems, vol. 25, pp. 1097-1105, 2012.

Liu, C., Linde, A.T., and Sacks, I.S., Slow earthquakes triggered by typhoons, Nature, vol. 459, no. 7248, pp. 833-836, 2009.

McCann, M.T., Jin, K.H., and Unser, M., Convolutional neural networks for inverse problems in imaging: A review, IEEE Signal Processing Magazine, vol. 34, no. 6, pp. 85-95, 2017.

Moehle, J., Seismic design of reinforced concrete buildings, McGraw-Hill Education, 2015.

Montgomery-Brown, E., Shelly, D.R., and Hsieh, P.A., Snowmelt-triggered earthquake swarms at the margin of long valley caldera, california, Geophysical Research Letters, vol. 46, no. 7, pp. 3698-3705, 2019.

Münchmeyer, J., Bindi, D., Leser, U., and Tilmann, F., Earthquake magnitude and location estimation from real time seismic waveforms with a transformer network, Geophysical Journal International, vol. 226, no. 2, pp. 1086-1104, 2021.

Perol, T., Gharbi, M., and Denolle, M., Convolutional neural network for earthquake detection and location, Science Advances, vol. 4, no. 2, p. e1700578, 2018.

Priestley, M.N., Seible, F., and Calvi, G.M., Seismic design and retrofit of bridges, John Wiley \& Sons, 1996.

Scholz, C.H., Tan, Y.J., and Albino, F., The mechanism of tidal triggering of earthquakes at mid-ocean ridges, Nature communications, vol. 10, no. 1, pp. 1-7, 2019.

Valentine, A.P. and Trampert, J., Data space reduction, quality assessment and searching of seismograms: autoencoder networks for waveform data, Geophysical Journal International, vol. 189, no. 2, pp. 1183-1202, 2012.

Yoon, C.E., O'Reilly, O., Bergen, K.J., and Beroza, G.C., Earthquake detection through computationally efficient similarity search, Science advances, vol. 1, no. 11, p. e1501057, 2015.

Zhang, X., Zhang, J., Yuan, C., Liu, S., Chen, Z., and Li, W., Locating induced earthquakes with a network of seismic stations in oklahoma via a deep learning method, Scientific reports, vol. 10, no. 1, pp. 1-12, 2020.

Zhou, Y.T., Chellappa, R., Vaid, A., and Jenkins, B.K., Image restoration using a neural network, IEEE transactions on acoustics, speech, and signal processing, vol. 36, no. 7, pp. 1141-1151, 1988.

Zoback, M.D. and Gorelick, S.M., Earthquake triggering and large-scale geologic storage of carbon dioxide, Proceedings of the National Academy of Sciences, vol. 109, no. 26, pp. 10164-10168, 2012. 